\title{
Reflexiones sobre los procesos de recuperación de los espacios públicos por medio de la participación activa: De la teoría a la práctica
}

\author{
Daniel Lira Sánchez
}

Ensayo

Invitado nacional

Profesional independiente

E-mail: dlirando.arq@gmail.com

Recibido: 13 de junio de 2018

Aprobado: 15 de noviembre de 2018
Daniel Lira Sánchez

Arquitecto graduado de la Universidad de Costa Rica. Director del estudio de arquitectura Dlirando Arquitectura. Consultor de diseño y presupuestario en proyectos arquitectónicos y urbanos, antiguo director ejecutivo de Asociación Semillas: Arquitectura en Comunidad. Énfasis en gestión territorial y de espacio público, desarrollo comunitario, diseño urbano, formulación y gestión de proyectos. Capacitador en talleres de activación urbana, de arquitectura y urbanismo emergente.

\section{Resumen:}

El presente ensayo expone las reflexiones del autor en torno a posturas teóricas relacionadas con el Derecho a la Ciudad, la gestión de los espacios públicos y los procesos sociales comunitarios y como estas se traducen a una práctica cotidiana para la producción de los espacios públicos comunitarios desde la gente. Repasa los conceptos de participación ciudadana, participación activa, organización comunal, procesos sociales, aprendizaje colectivo, derecho a la ciudad, espacio social, entre otros. Esto se contrapone con lo realizado en tres proyectos o procesos sociales que buscan la recuperación de sus espacios públicos comunitarios por medio de la participación de las comunidades en miras a un desarrollo comunal más integral.

Palabras Clave: espacio público; participación activa; derecho a la ciudad; producción del espacio; recuperación de espacios públicos.

Reflections on the processes of recovery of public spaces through active participation: From theory to practice

\section{Abstract:}

This essay exposes the thoughts of the author regarding the theory about the right to the city, public spaces management, and social community processes. It also shows how they render to a day to day practice on the production from the people of these communal, public spaces. This reviews the concepts of citizen participation, active participation, community organization, social processes, collective learning, right to the city, social space, among others. This constrasts with what has been done in three projects or processes to recover their public spaces through the involvement of the communities towards a more integrated community development.

Keywords: public space; active participation; right to the city; productions of space; public spaces recovery. 


\section{Premisa:}

La participación activa como mecanismo para el desarrollo de las comunidades por medio de la recuperación de sus espacios públicos.

\section{Introducción}

omo profesionales en arquitectura y urbanismo, muchas veces consideramos que la producción del espacio es solo tarea nuestra, razón por la que diseñamos estos espacios como agentes externos a las comunidades y sus contextos específicos. El filósofo Henri Lefebvre (2013) plantea que el espacio es el resultado de las acciones, las prácticas, las relaciones y las experiencias sociales, pero al mismo tiempo es parte de estas. De esta forma, se podría decir que el espacio público es un producto social, por lo que la producción del espacio público resultaría de una construcción colectiva desde los habitantes. Así, los habitantes serían productores del espacio y al mismo tiempo serían gestores que a la vez formarían parte del espacio que producen. En consecuencia, la producción del espacio es un proceso. Desde un enfoque como el de la cogestión social del hábitat como lo plantean Morales y Obando (2016), la producción de espacio es mucho más que la construcción física de la infraestructura que genera el espacio. También es la construcción de los vínculos simbólicos que cada individuo crea por medio de las dinámicas sociales que suceden gracias al intercambio entre habitantes en donde estos procesos se nutren a sí mismos.

En el contexto internacional, la Organización de las Naciones Unidas proclama en el 201517 objetivos para el desarrollo sostenible como una guía para alcanzar las metas propuestas en la Agenda 2030. El objetivo de desarrollo sostenible número once se enfoca en las ciudades, las comunidades sostenibles y en como aún estas enfrentan grandes retos en temas relacionados a espacios públicos y verdes. La ciudad está descuidada. Si se piensa que la ciudad es un tejido urbano con identidad simbólica en donde ese simbolismo nace de la manera en que los habitantes se apropian de los lugares, entonces cada uno de ellos en distintas formas y niveles son los que crean, los que construyen la ciudad en la que viven:

El derecho a la ciudad ... es mucho más que el derecho de los individuos o los grupos a acceder a los recursos que la ciudad encarna: es el derecho a cambiar y a reinventar la ciudad según el deseo en nuestros corazones... La libertad de hacernos y rehacernos a nosotros mismos y a nuestras ciudades es ... uno de los más preciosos si bien más descuidados de entre los derechos humanos. Quizás por esta razón intuitiva, la ciudad ha sido el foco a lo largo de la historia de una inmensa efusión de deseos utópicos de futuros más felices y tiempos menos alienantes. (Harvey,2014, p. 282)

Entonces, ¿cómo se reinventan las ciudades? ¿Cómo hacemos valer el derecho a la ciudad? ¿Hacia dónde se debe orientar el cambio de las ciudades? Hoy en día, la mayor parte de la inversión en las ciudades se concentra en los espacios destinados únicamente para vehículos, dejando en un segundo plano a la ciudadanía que vive la ciudad desde la peatonalidad, con espacios públicos que tienen horarios de acceso restringido con poca o nula inversión en mantenimiento o creación de espacios públicos como aceras en mal estado o inexistentes, entre otros. Es necesario fortalecer el sentido de comunidad por medio de los procesos de recuperación de los espacios públicos y permitiendo restablecer el sentimiento de colectividad dentro de las ciudades. Entonces, ¿hacia dónde se debe orientar el cambio de nuestras ciudades? Viviéndola, cada uno, desde lo colectivo, desde las comunidades y desde los espacios públicos. Alzando la voz, conversando con los vecinos sobre oportunidades y posibilidades para nuestra ciudad.

El presente ensayo se plantea en dos partes. La primera consta de la exposición de un marco conceptual sobre el derecho a la ciudad, los espacios públicos, la recuperación de estos y sobre la participación ciudadana. La segunda parte presenta tres procesos comunitarios participativos en los que se busca llevar la teoría expuesta a la práctica con el fin de recuperar los espacios públicos de estas comunidades. 


\section{Marco conceptual}

Como se menciona anteriormente, se parte bajo la perspectiva del derecho a la ciudad. Con el fin de establecer un panorama claro, se recurre a los apuntes realizados por $\mathrm{F}$. Molano (2016) acerca de las transformaciones planteadas por Harvey, Soja y Carlos a la definición inicial de Lefebvre. Este constituye el derecho a la ciudad como el derecho de los habitantes a construir, decidir y crear la ciudad y Harvey, Soja y Carlos, lo asumen como "un marco de comprensión para el estudio de los problemas socio-espaciales urbanos" (Molano, F 2016, p.4).

El autor resume las posturas trasformadoras del derecho a la ciudad de la siguiente forma:

Harvey: "posibilidad social de lucha y teoría anticapitalista, que haga del territorio urbano un escenario de construcción de alternativas de apropiación y participación política y espacial."

Soja: "articula formas de resistencia étnica, de género y cultural, como expresión de la diversidad de experiencias urbanas."

Carlos: "recuperar el espacio público urbano apropiado por el capital financiero."

Estas tres reflexiones permiten visualizar una aproximación a la teoría sobre el derecho a la ciudad con la práctica a través de los procesos de recuperación de los espacios públicos comunitarios por medio de la participación activa. Primero, se observa como una de las formas de hacer valer este derecho es mediante las alternativas de apropiación y participación desde los habitantes articulados bajo la expresión de sus diversas experiencias en un plano de equidad participativa. Por último, trae a la discusión el tema del impacto de la economía en los espacios públicos:

La ciudad es ante todo el espacio público, el espacio público es la ciudad. Sin espacio público potente, integrador socialmente, articulador físico y simbólicamente, la ciudad se disuelve, ... la reducción de las desigualdades y la supremacía de la solidaridad y la tolerancia como valores ciudadanos se ven superados por la segregación y la codicia, por el egoísmo y la exclusión. (Borja, 2012, p. 1)

Todo lo anterior es posible a través de procesos sociales como mecanismos de fomento de la participación activa y herramientas de capacitación, vínculo y fortalecimiento comunitario. Además, estos crean instancias de intercambio transdisciplinario en donde los habitantes se pueden ver favorecidos de la experticia de los facilitadores y de otros gestores, así como estos otros pueden beneficiarse de la experiencia empírica, el conocimiento cotidiano y de su contexto de los vecinos y vecinas. La ciudadanía debe recuperar el rol en la planificación, apropiación y gestión no solo de los espacios públicos, sino también de la ciudad misma, proyectando soluciones propias para las necesidades inmediatas que cada comunidad tenga en relación con su espacio público.

Recordando el agenciamiento espacial planteado por Awon, Schneider y Till en el 2011, el espacio es un producto social, al igual que lo define Lefebvre. Esto recalca que, dentro de la práctica profesional, los arquitectos y planificadores deben tomar en consideración "sus creaciones son anticipaciones de una futura relación social, no solamente un producto estético. Por lo que los espacios comunes creados deben corresponder a los espacios sociales (existentes) que están cargados de dinámicas de poder, empoderamiento, interacción, aislamiento, control y libertad" (Awan et al. citado en Suárez, 2018, p. 30). De esta forma "un fanzine, un blog, mapas y el soporte técnico son tan arquitectura como lo es un edificio. Lo que los diferencia es que producen y diseminan los saberes espaciales de una forma en la que está conscientemente abierto a otros" (Awon, et al, 2011, p. 65).

Desde el quehacer como profesionales en arquitectura y urbanismo es necesario dejar el rol de productor y producto como enfoque de diseño únicamente, ya que en algunos casos los productos suelen resultar en diseños de espacios áridos, poco apropiables, rígidos y privatizados. Probablemente, estos espacios estén diseñados a la perfección, pero no están conectados con los habitantes de donde se encuentran. Muchas veces se habla de cederle espacio a la ciudad, lo que en teoría está bien, pero se debe pensar más en darle ese espacio a las personas que lo habitan primero y por consiguiente a la ciudad. 
En relación con lo anterior, se visibiliza la necesidad de que como sociedad se inicie un proceso educativo de formación acerca del valor de los espacios públicos para la cogestión social de la ciudad, en donde se discutan temas de seguridad ciudadana, apropiación, mejoramiento barrial, organización comunitaria, movilidad, gestión de residuos y gestión de riesgos, así como conocimientos sobre la gestión de los espacios públicos propiamente; de manera que esto permita regenerar la cultura ciudadana, estrechar vínculos, minimizar diferencias y enriquecer las dinámicas vecinales. Los procesos sociales son una inmensa multitud de interacciones de los seres humanos, actuando en combinaciones 0 en grupos. Estos traen consigo gran variedad de beneficios para las personas participantes, siendo el aprendizaje en colectivo uno de los principales.

En la actualidad la disciplina del aprendizaje en equipo está a punto de lograr grandes innovaciones, pues gradualmente estamos aprendiendo a 'practicar'. Se están desarrollando dos 'campos de práctica': El primero concierne a la práctica del diálogo, de modo que un equipo empiece a desarrollar aptitudes conjuntas para alentar un cociente intelectual de equipo que supere el cociente intelectual de los individuos. El segundo concierne a los 'laboratorios de aprendizaje', donde el aprendizaje en equipo afronta la dinámica de realidades complejas. (Gutiérrez, 2006, p.3)

Las oportunidades de formación ligadas a estos procesos permiten mejorar las relaciones entre las personas habitantes, consensuar sus opiniones y orientar el aprendizaje a resolver las necesidades colectivas reales que enfrentan las distintas comunidades. Esta colectividad abre las puertas a la organización comunitaria y a la participación ciudadana activa como una herramienta de trabajo para el desarrollo comunitario propio, así como al crecimiento profesional como arquitectos y urbanistas, el cual permite ampliar la visión de lo que verdaderamente es la arquitectura.

La participación ciudadana constituye el involucramiento de un conjunto de habitantes en la toma de decisiones, de manera que los gobiernos o entes representativos cuenten con un respaldo para el planteamiento de proyectos e iniciativas. Camacho (2017), explica cómo este concepto obedece al "principio de que las y los gobernados tomen decisiones activas como parte del gobierno, es una idea que, en teoría, es respaldada por una gran mayoría, sin embargo, es un momento de negociación entre figuras de poder y un espacio de conflicto" (p. 51). Más adelante, la misma autora hace referencia a CYTED (2004), cuando se refiere a la importancia de diferenciar entre los diferentes tipos de participación, así establece que "se debe considerar tanto la participación ciudadana como la participación institucional y la técnica/profesional, entendiendo que los niveles de participación de estas instancias varían de acuerdo con procesos de diálogo y entendimiento recíproco, sin poder generalizar todos los procesos en una misma categorización genérica o ambigua" (CYTED citado en Camacho, p. 57).

A partir de estos postulados, se considera primordial definir y delimitar el concepto de participación activa. Para esto, se toma la definición propuesta por Camacho, en donde la participación activa "implica un acercamiento constante a lo largo de o en distintas etapas específicas de un proceso, así como un rol contundente en la toma de decisiones, de emitir voz y voto. La participación activa requiere de un compromiso expreso, práctico y efectivo por parte de quien la ejerce. Existe un interés individual y colectivo por el mejoramiento de las condiciones de habitabilidad de una comunidad" (2017, p. 185).

En términos de la escalera de participación propuesta por Sherry Arnstein en 1969, la participación activa aparece en el quinto escalón denominado conciliación. Este se refiere a un "nivel de 'participación' activa con cierto grado de influencia en la toma de decisiones para el planteamiento, gestión y desarrollo de los procesos" (Camacho, 2017, p. 55). Asimismo, surge el sexto escalón designado como asociación. Este responde a un "mecanismo de decisión conjunta y colectiva, entre el equipo facilitador y/o gestor, las autoridades institucionales y la comunidad, procurando la participación real y efectiva de todas las partes involucradas" (Camacho, 2017, p. 55). En cuanto a los últimos dos escalones propuestos por Arnstein, poder delegado y control ciudadano, estos se plantean en términos de representatividad y movimientos organizados que no necesariamente garantizan una participación activa en torno a los procesos relacionados con espacios públicos. 
Camacho plantea dos eslabones más a la escalera de participación planteada por Arnstein. Estos nuevos escalones contemplan las mayores expresiones de participación activa para los procesos sociales en torno a los espacios públicos comunitarios. En el noveno peldaño está la cogestión, la cual se presenta como un "proceso colaborativo de integración entre las bases comunitarias organizadas, apertura a la participación de nuevos integrantes de la comunidad, representación de instituciones públicas (gobierno local o central), empresas privadas, instituciones académicas, organizaciones no gubernamentales y civiles, entre otras" (Camacho, 2017, p. 58). El último escalón lo define como autogestión en donde la "comunidad demuestra una capacidad de organización orgánica", así como "capacidad de autonomía comunitaria en la toma de decisiones" (Camacho, 2017, p. 58).

La participación ciudadana se plantea únicamente en relación con la institucionalidad y uno desde una perspectiva de participación activa como se define anteriormente. A lo largo del país existen esfuerzos desde la gente que luchan por mejorar sus comunidades de manera activa y continua, participando con sus vecinos y organizándose entre sí sin necesidad de una figura institucional. Estos esfuerzos permiten la integración de los distintos sectores, de la academia y de organizaciones sin fines de lucro.

Desde la gestión institucional tradicional, el planteamiento se presenta como un simple requisito o idea y no llega a ejecutarse ni a vincularse con los habitantes. Tomando como ejemplo el caso de la Municipalidad de San José, esta cuenta con la Política de Participación y Corresponsabilidad Ciudadana, cuyo objetivo es "fomentar una cultura de participación y corresponsabilidad ciudadana, en el cantón de San José, con el fin de ampliar la democracia y mejorar la gestión municipal" (Municipalidad de San José, 2015, p. 14). Desde un punto de vista institucional, se pretende ver la participación ciudadana como un componente más de la democracia representativa que se nutre de los recursos de los sectores sociales, así como de los recursos y capacidades institucionales.

Esta política espera ser un instrumento formal programático que articule el quehacer municipal y contribuya a la democratización, considerando las demandas e intereses de los habitantes. Sin embargo, la realidad es que esta política no logra aplicarse ni llevarse a la práctica, ya que en la misma no se plantean mecanismos para establecer el contacto y acercamiento con la ciudadanía, así como los procesos y proyectos para propiciar la interacción de las personas vecinas en sus distintas etapas de planteamiento, gestión y ejecución. En este caso, desde la institucionalidad no existe un verdadero acercamiento a las comunidades y a los habitantes con necesidades comunitarias específicas. Los habitantes resultan ajenos a los planteamientos e iniciativas, en dónde las intervenciones resultan genéricas, por salir del paso o por compromiso y sin considerar las necesidades de las personas ni su contexto.

En contraste y como ejemplo de participación ciudadana activa está el caso de las comunidades de Las Flores y el Sitio en Caño Negro, donde lejos de una institucionalidad formal, la organización comunitaria les permitió a los habitantes de estas comunidades, de forma cogestionada, construir el rancho comunitario que necesitaban para formalizar sus esfuerzos colectivos en un espacio público; aprovechando la construcción de este espacio como la excusa para discutir problemáticas y oportunidades para ellos como comunidad organizada. En este caso, el proceso se vio enriquecido por la participación de colectivos estudiantiles, organizaciones sin fines de lucro y cooperación internacional como agentes de financiamiento y asesoría técnica.

Estos procesos tienen la posibilidad de convertirse en mecanismos de gestión que logren impulsar la apropiación de los espacios públicos y el desarrollo comunitario por medio de la integración de las personas que habitan en las distintas fases y posibilidades desde las dinámicas cotidianas de la ciudadanía y hasta una gestión institucional que les regrese el poder a las comunidades, buscando una cogestión social de la ciudad, en donde se establece una corresponsabilidad entre todas las partes y existe una articulación fuerte. Esto se logra desde la participación ciudadana activa y la organización comunal como lo menciona Morales y Obando (2016).

¿Cómo se logra el desarrollo comunal desde los habitantes? ¿Cómo se vincula la participación ciudadana al desarrollo comunitaria? ¿Será necesario replantear el modelo 
de organización institucionalizada y rígida, como es el caso de las asociaciones de desarrollo? ¿Qué herramientas y habilidades necesitan las comunidades para alcanzar sus metas colectivas?

Por medio de los procesos de recuperación de espacios públicos que integran la participación activa, se pueden gestionar esfuerzos que brinden a las comunidades, a sus líderes y lideresas, adultos, jóvenes y niñez, los insumos para que ellos mismos planteen, gestionen y ejecuten los proyectos de infraestructura y desarrollo comunitario que necesitan junto con la ayuda y financiamiento de la institucionalidad, garantizando una mejor distribución de los recursos públicos. De esta manera, las organizaciones vecinales tienen la potestad de dictar cuáles son las mejoras en temas de accesibilidad, manejo y canalización de aguas, mantenimiento e inversión de mobiliario urbano, arborización y cobertura vegetal y mejoramiento barrial, así como los diferentes tipos de actividades que los espacios públicos les permitan realizar. "Una transformación de la sociedad supone la posesión y la gestión colectivas del espacio mediante una intervención constante de los "interesados", con sus múltiples, diversos y contradictorios intereses" (Lefebvre citado en Molano, 2016, p. 8).

En este punto es vital aclarar qué se define como recuperación de espacios públicos. Para esto se recurre nuevamente a los apuntes de Harvey sobre el derecho a la ciudad. Este debe "plantearse, no como un derecho a lo que ya existe, sino como un derecho a reconstruir y recrear la ciudad (...) con una imagen totalmente diferente" (Harvey citado en, 2016, p. 10). Además, defiende el concepto de las "prácticas ciudadanas insurgentes 1 " como un medio que permite plasmar las utopías que pretenden convertir al territorio en un "escenario de construcción de alternativas espaciales, que restrinjan los efectos urbanos de la apropiación capitalista del espacio" (Molano, 2016, p. 9), como lo ha sido la privatización del espacio público, gentrificación o la dotación de equipamiento urbano publicitario tipo 'mupis' o vallas publicitarias, así como a la idea por parte de las instituciones de que los espacios públicos son homogéneos y libres de conflicto.

Para el presente ensayo, se toma como recuperación de espacios públicos todos los esfuerzos desde la ciudadanía por (re)apropiarse de sus espacios públicos por medio de procesos sociales que permitan cambiar, recrear o reconstruir estos espacios en sitios de intercambio e interacción entre las personas habitantes, en donde estos cambios pueden surgir desde las "prácticas ciudadanas insurgentes" o alternativas lejanas de la formalidad de la institucionalidad gubernamental, haciendo así valer el derecho a la ciudad.

Laarticulaciónintersectorialentreinstitucionespúblicas, empresaprivada, organizaciones sin fines de lucro y la ciudadanía en general es vital para llevar a cabo estos procesos, en donde se garantice un intercambio de saberes interdisciplinario y donde cada participante tiene la posibilidad de expresar y compartir sus intereses, necesidades, experiencias, experticia, conocimiento técnico, entre otros. De esta forma, se genera un ciclo de aprendizaje, diálogo y fortalecimiento del sentimiento de comunidad. Es importante mencionar que la escala de los procesos no resulta trascendental ni la cantidad de habitantes que colaboren. Lo primordial es la trascendencia de estos dentro de las comunidades y los espacios públicos, en términos de apropiación, empoderamiento, activación de la ciudad y participación activa.

A partir de todo lo mencionado anteriormente, se podría decir que la cogestión social

1 Como anota Molano (2016), Harvey toma el concepto de "ciudadanía insurgente" de James Holston, para quien estas expresiones ciudadanas surgidas en las periferias urbanas populares, partiendo de los marcos legales vigentes, "empoderan, parodian, descarrilan o subvierten las agendas estatales", amplían las nociones de ciudadanía y abren el horizonte político hacia formas propias y autónomas de construcción política (Holston, 2008, p. 3-35). del hábitat, la ciudad, las comunidades y de los espacios públicos es permanente y continua. Además, esta no se limita a procesos de recuperación de espacios públicos, sino que abarca también aspectos de planificación, movilidad, vivienda, educación, cultura, entre otros. El tejido del sentimiento de comunidad surge de manera orgánica, donde los enlaces y vínculos se dan de forma espontánea y natural. De igual forma, sucede con el sentido de apego y pertenencia en los espacios públicos por parte de las personas habitantes, los cuales van forjándolo día a día en una cotidianidad positiva. 


\section{Puesta en prática}

A continuación, se presenta una serie de procesos que pretenden mostrar cómo se pueden llevar estos postulados teóricos, expuestos anteriormente, a la realidad. Esto por medio de la labor transdiciplinaria de profesionales en arquitectura, economía, administración pública y con apoyo de profesionales en sociología, antropología e ingeniería que han trabajado en conjunto con comunidades en distintas partes del país, tanto en zonas urbanas como rurales.

Todo empieza con una iniciativa de cambio. La mayoría de las ocasiones, esta se origina desde las lideresas o líderes comunales en donde ellos visibilizan y exponen una necesidad que debe ser atendida para el beneficio de todas las familias de la comunidad, así como vecinas de áreas aledañas. Ahora, es importante recordar que la gestión de los espacios públicos es una tarea que por ley le corresponde a las municipalidades, sin embargo, la mayoría de los municipios no poseen la capacidad de gestión para atender las necesidades de las comunidades, esto según el Índice de Gestión Municipal realizado por la Contraloría General de la República, en donde el rubro de Parques y Obras de Ornato es la peor calificada a nivel nacional ${ }^{2}$. Razón por la cual es imprescindible la articulación intersectorial.

\section{Comunidades de Los Olivos y Los Geranios}

El primer caso inicia a finales del 2016, cuando Marilyn Barrientos, vecina de Los Olivos en Paso Ancho, San José, contactó a Asociación Semillas ${ }^{3}$ con la intensión de buscar apoyo en la recuperación del parque ubicado entre su comunidad y la comunidad de Los Geranios. Ella junto con un grupo de líderes y lideresas de ambas comunidades decidieron formar el Comité Pro Parque Los Olivos-Los Geranios con el objetivo de luchar por su derecho a tener espacios urbanos verdes dignos, aptos para la recreación de los vecinos y vecinas, tanto jóvenes como adultos, así como infantes y adultos mayores.

"El proyecto del parque surgió hace un año y medio, -dos años-, donde dos vecinos iniciamos con la esa espinita de que sería volver a recuperar un espacio que estaba completamente perdido." (Marilyn Barrientos, comunicación personal, noviembre 11, 2017)

De esta forma, inició un proceso de cogestión de espacio público por parte del Comité Pro Parque Los Olivos-Los Geranios y Asociación Semillas. A lo largo de este proceso, se han organizado diferentes actividades como clases de zumba, festivales deportivos, campañas de reciclaje, bingos, conciertos de música, actividades de mantenimiento y limpieza, muchas de estas con el fin de recaudar fondos para las mejoras del propio parque. Además, como parte del acompañamiento técnico, dentro del proceso de cogestión, Asociación Semillas facilitó el proceso de diseño del parque, en donde se incorporaron varios talleres de diseño participativo, talleres de validación y jornadas de trabajo, las cuales se denominaron como "Manos a la Obra", en donde la comunidad realiza la construcción de mejoras de pequeña escala, como la instalación de mobiliario urbano, siembra y mantenimiento de áreas verdes y pintura de murales. De esta manera, se busca integrar el involucramiento ciudadano en el desarrollo comunitario, la incorporación de la corresponsabilidad entre vecinos y el arraigo y apropiación del espacio público. Este tipo de iniciativas, a cargo de la ciudadanía en búsqueda de una mejora de su barrio, podrían considerarse como prácticas ciudadanas insurgentes a las que se refiere Harvey, siendo estas estrategias alternativas para reconstruir la ciudad, y por consiguiente, formas de luchar por el derecho a la ciudad.

"Ha sido un proceso bastante difícil, desagraciadamente, por ejemplo por parte de la Municipalidad hemos tenido apoyo, pero no quizás el apoyo que nosotros quisiéramos... Entonces, ha sido ir buscar, seguir tocando puertas. Nos hemos reunido como comunidad, entonces hacemos reuniones en mi casa, en el salón comunal o en la casa de algún vecino, hacemos actividades... ahí hemos ido, poco a poco, generando los recursos para poder hacer, de a poquito, unos cambios bien importantes para el parque." (Marilyn Barrientos, comunicación personal, noviembre 11, 2017) ${ }^{4}$ 
Figura 1: Proceso de diseño. Actividad: ¿Un parque seguro es?. Autoría: Asociación Semillas.
$5 \quad$ Entrevista con Silvia Camacho Quesada co-coordinadora del proyecto. Junio 2018.

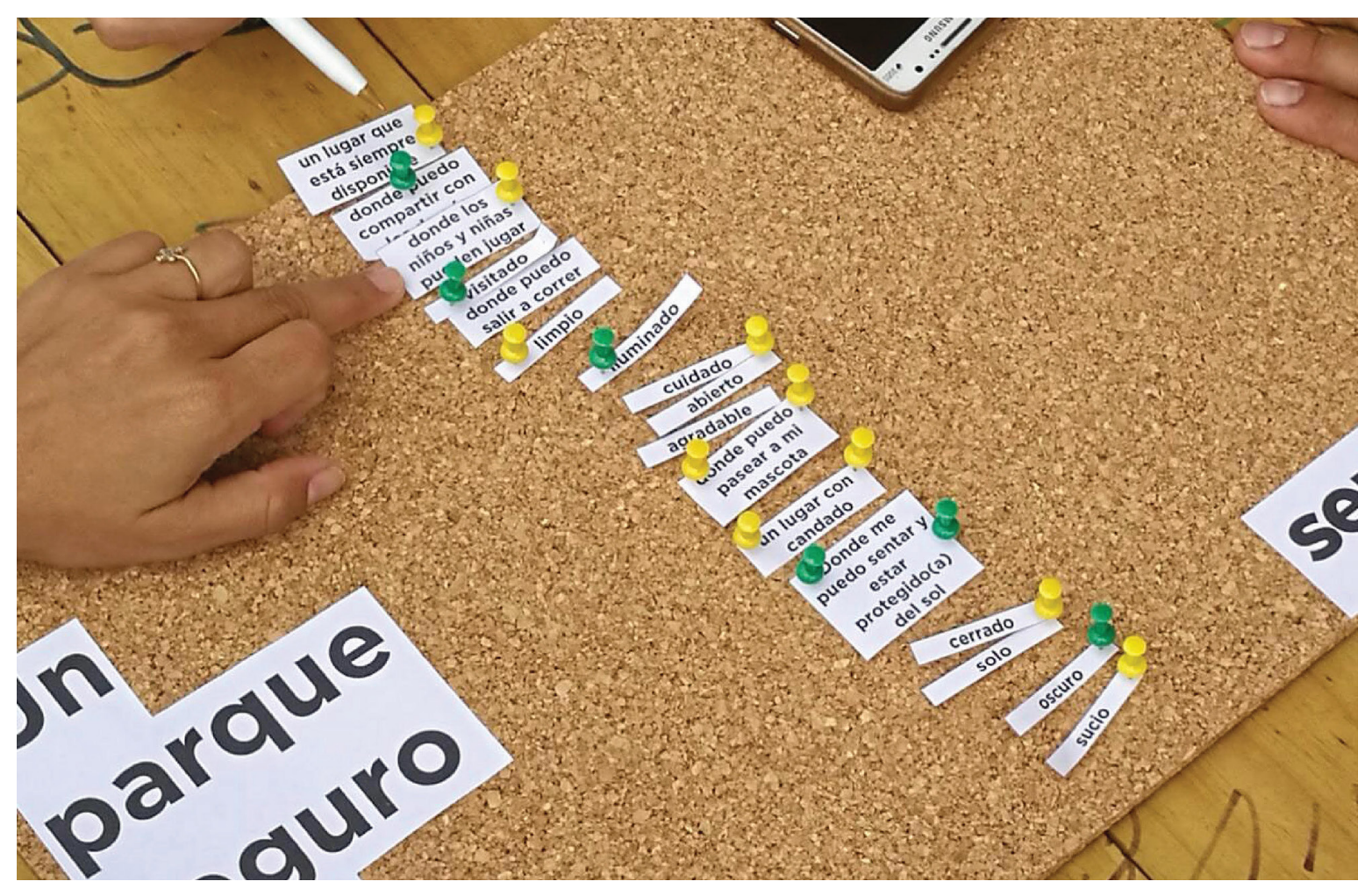

Propiamente, el proceso de diseño incorpora diversidad de visitas, talleres, actividades y jornadas de construcción activa, todo esto en conjunto con las personas vecinas de la comunidad. Los primeros talleres cuentan con un enfoque de diagnóstico. La arquitecta Silvia Camacho, co-coordinadora del proyecto, comenta como en las primeras convocatorias, el proceso se concentró en "el fortalecimiento del tejido comunitario y la gestión social, el reconocer personas con un rol de líderes que tuvieran roles estratégicos y que se conocieran entre sí, al ser de barrios distintos" (Silvia Camacho, comunicación personal, junio 21,2018$)^{5}$, en una etapa inicial, la cual ella conceptualiza como talleres de redes. La fase siguiente abordó el tema de las necesidades del espacio físico, como se muestra en la Figura 1. En estos talleres se profundizó en los conceptos de seguridad, parques, sus necesidades y como describen e imaginan a su comunidad, menciona Camacho. Esta fase se trabajó bajo en concepto de talleres de reconociendo mí comunidad.

A partir de estos talleres se construyeron pautas y lineamientos de diseño a cargo de los diseñadores en una etapa no tan participativa, si no que técnica, en donde se concentran en volver tangible todas las ideas, propuestas, necesidades y expectativas expresadas por la comunidad en una propuesta de diseño de anteproyecto preliminar. Este diseño entra en una nueva etapa de validación con los vecinos y vecinas, donde la comunidad hace comentarios y sugerencias específicas de la propuesta de diseño. En este punto, describe Camacho como "entran variables de conductas y comportamientos que pasan en el lugar, como por ejemplo, los accesos secundarios al parque -que estaban antes cerrados- y estrategias, inclusive, de cómo gestionar las terrazas y jardines, la luz, el parque de los perros y este tipo de temas. Entonces, en el proceso de validación, las personas empiezan a aportar ideas de cómo gestionar y cómo se puede intervenir para que esto se construya, desde la misma cotidianidad de la comunidad." (Silvia Camacho, comunicación personal, junio 21, 2018)

Desde el planteamiento del proyecto, las co-coordinadoras establecen como prioridad alternar los talleres, reuniones y jornadas de trabajo en campo como se muestra en la Figura 2. Esto porque si el proceso se centralizaba, únicamente al inicio en reuniones con la comunidad sin que los vecinos y vecinas pudieran observar un trabajo palpable y que se empezaran a ver mejoras en el parque, se iba a perder el entusiasmo por parte de las personas participantes. De esta manera, se buscaba propiciar una participación por parte de los vecinos y vecinas en distintos grados, en donde hubo gente con un mayor liderazgo y encargadas de la gestión del proyecto, así como personas participantes más esporádicas en las jornadas de trabajo en sitio. El proceso pretendía incorporar un rango de participación activa acorde con los intereses propios de sus habitantes, así como la disponibilidad de tiempo con que estos contaban. Esto permite reflexionar si los postulados teóricos de la escalera de participación deben contemplarse o referirse en torno a los procesos como un todo o si lo más acertado sería considerar la participación activa como un elemento propio de cada participante de dicho proceso. 
Figura 2: Sesión de "Manos a la Obra". Autoría: Asociación Semillas.

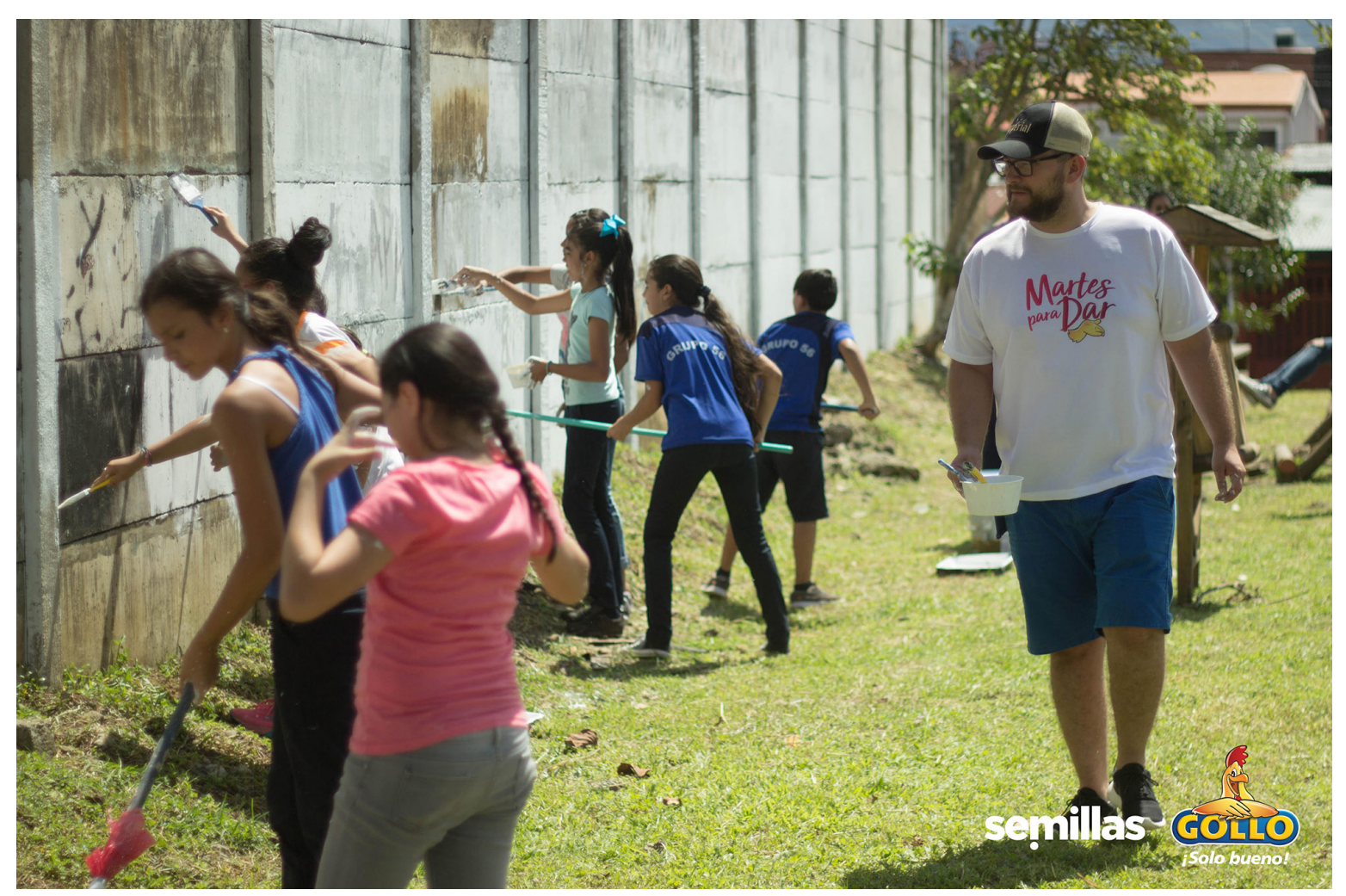

Camacho se refiere a lo anterior de la siguiente forma:

Es muy importante, alternar los momentos de encuentro con la comunidad. Permite tener una visión más estratégica de lo que se está haciendo y recibir insumos en distintos momentos y con diferentes núcleos de personas que están interviniendo. Hay personas que son más participantes estratégicas, otros más de convocatoria y otros más de usuarias que no siempre pueden estar a lo largo de cada paso, pero si asisten y participan en los eventos que son en el parque y eso es muy importante. (Silvia Camacho, comunicación personal, junio 21, 2018)

Este proceso comunitario incorpora el tema del derecho a la ciudad y la recuperación de espacios públicos por medio de la implementación de las sesiones de manos a obra, en donde la comunidad reconstruye su propio espacio público. Como se menciona anteriormente, la participación activa en procesos sociales relacionados con los espacios públicos comunitarios no solo permitiría más que la construcción física del hábitat, por ejemplo, espacios de reunión, canchas multiusos, parques para perros, áreas de máquinas de ejercicios, ranchos comunitarios, huertas comunitarias, áreas de acceso, entre muchos otros, sino que también permitiría la producción social de dichos espacios, generando vínculos comunitarios fuertes, fomentando la base organizativa de las comunidades y las relaciones inter-vecinales.

\section{Comunidad de Lomas del Río}

El segundo ejemplo da inicio en el 2018, momento en el que Urbarium ${ }^{6}$ inicia un proceso en la comunidad de Lomas de Río en Pavas, luego de un acercamiento que tuvieron al intervenir la Cancha Holanda, espacio público en barrio Bribri. Esto les permitió vincularse con mayor fuerza con la organización comunal, específicamente con la Asociación de Desarrollo de Lomas I y la Asociación de Desarrollo Específica Pro-Mejoras y Obras Comunales, con miras de recuperar unos de los espacios públicos más emblemáticos y simbólicos de la comunidad. Se establecen como ejes primordiales de trabajo a realizar en conjunto la recreación y prevención de la violencia, vinculando el arte como medio para generar empoderamiento y participación comunitaria.

En la etapa de diagnóstico, según comenta K. Cubillo, gestora de proyectos para la organización, la comunidad presenta "problemáticas relacionadas con la poca participación comunitaria, el mal manejo de basura y residuos sólidos, los altos índices de violencia lo que ha generado una alta estigmatización social comunitaria." (Karen Cubillo, comunicación personal, octubre 25,2018 ). Con el fin de contribuir al desarrollo comunitario y contrarrestar esta estigmatización social, se plantea como objetivo "reactivar la cancha de la comunidad de Lomas I (conocida como la Cancha Azul), mediante la vinculación del arte, con el fin de promover la participación comunitaria y la apropiación del espacio público para la recreación y la interacción social." (Karen Cubillo, comunicación personal, octubre 25, 2018). De esta forma, desde su inicio el proceso 
Figura 3: Sesión de construcción. Autoría: Urbarium.

Figura 4: Actividad recreativa como parte de la inauguración de la Cancha Azul. Autoría: Urbarium.

$7 \quad$ Testimonio de Eugenia Nuñez de la Asociación de Desarrollo de Lomas del Río, Pavas. busca introducir y fortalecer la práctica de participación activa entre los habitantes de la comunidad, como se observa en la Figura 3.

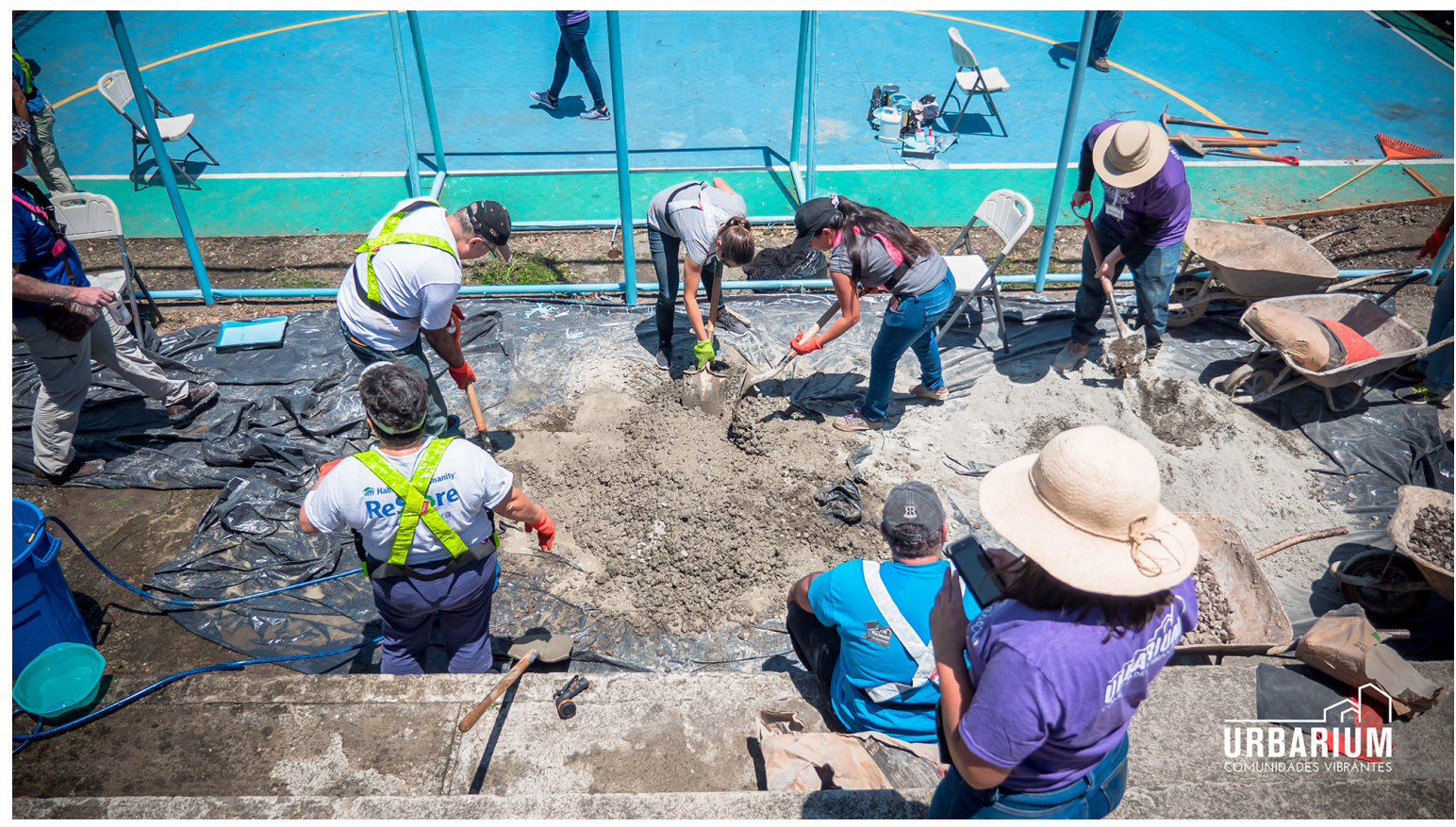

"Es un espacio (la Cancha Azul) utilizado totalmente por la juventud y la niñez, en donde ellos pueden desarrollar sus habilidades y encontrar un espacio seguro y totalmente habilitado para practicar deporte." (Eugenia Nuñez, comunicación personal, junio, 2018)

Similar a la metodología empleada por Asociación Semillas, Urbarium incorpora en su proceso social jornadas de trabajo en el espacio público de la comunidad. En este caso, las tareas de mejora han sido acompañadas por el apoyo de organizaciones internacionales como ONG Hábitat para la Humanidad Latinoamérica y El Caribe y el Centro Panamericano de Idiomas. En estas actividades han participado más de 28 niños, niñas y jóvenes de la comunidad. A estos se les han unido alrededor de 109 jóvenes extranjeros por medio de los programas de voluntariado, lo cual ha incentivado el intercambio cultural y la (re)apropiación de la cancha con las actividades recreativas.

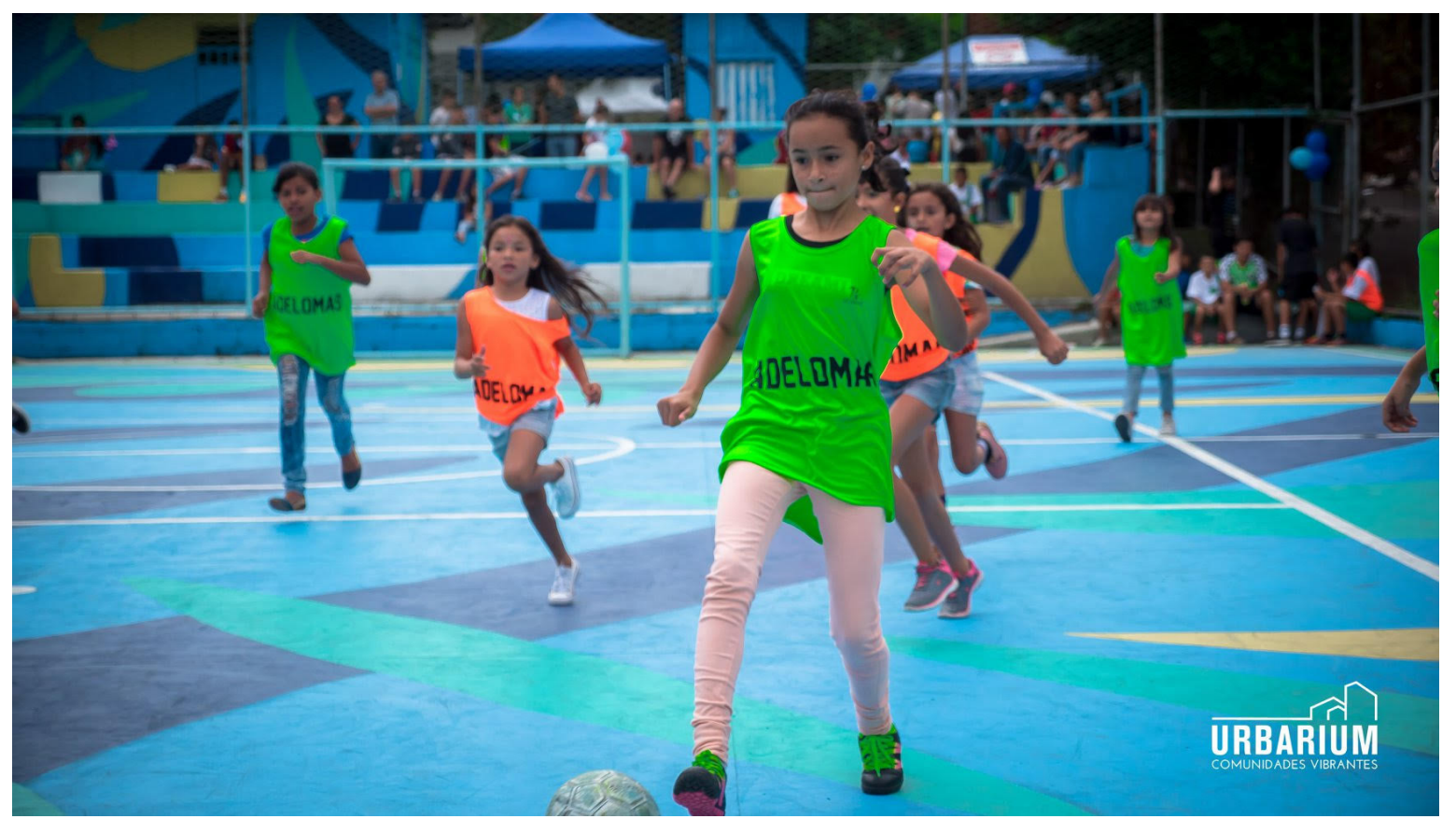

El trabajo continuo con el grupo de jóvenes de la comunidad ha ocasionado un surgimiento y fortalecimiento de liderazgo entre ellos, los cuales, según menciona Cubillo, representan la acción comunitaria del barrio. A partir del modelo de gestión comunitaria participativa que la organización ha impartido y de las sesiones de trabajo lúdicas, se busca promover las capacidades personales y colectivas que fomentan la "generación de agentes de cambio" que se involucran en las mejoras en sus espacios públicos y hacen valer su derecho a la ciudad. Este grupo de jóvenes se consolidó y fue nombrado por ellos mismos como Los Imaginadores.

Dentro de las actividades planteadas dentro del marco del proceso se realizó un Festival Recreativo. Este vinculó a organizaciones comunitarias como la Asociación de Desarrollo Integral de Lomas I, gobierno local, instituciones como el PANI, el Ministerio de Salud y la Fuerza Pública. Estas actividades procuraban la promoción de las características recreativas, deportivas y artísticas de los habitantes de la comunidad por medio de visibilidad a las artesanías y la cultura local como se muestra en la Figura 4. 
En este caso, la incorporación del arte como forma para involucrar a la comunidad en la recuperación de la cancha resultó un medio interesante para hacer valer el derecho a la ciudad. Cada proceso social es distinto y responde al ritmo de cada comunidad en términos de participación. Asimismo, las actividades, reuniones y sesiones de trabajo se acoplan a las necesidades colectivas y temáticas por trabajar.

\section{Comunidad de Sevilla}

El último caso inicia a finales del 2017, cuando Shirleny Vargas, lideresa de la comunidad de Sevilla en Dulce Nombre de Coronado, decide contactar a Asociación Semillas con el fin de buscar apoyo técnico en la gestión para la recuperación de dos parques de su comunidad. Ella, junto con Marisol Alvarado, han liderado los esfuerzos de recuperación de este espacio público desde hace más de tres años, momento en que se consolidó en el Comité Pro Mejoras del Parque Sevilla 1 y 2. Durante este tiempo, el comité ha estado trabajado tanto en mejoras pequeñas como siembra de árboles y flores, así como pintura de murales con el fin de embellecer el parque, nuevamente estos esfuerzos iniciados desde la ciudadanía aluden a las antes mencionadas prácticas insurgentes. Además, han articulado con la Municipalidad de Coronado la solicitud y aprobación de una partida específica para el mantenimiento de las mallas existentes.

"Nosotras empezamos con una idea, teníamos un parque sin nada, era un parque que no tenía absolutamente nada, más que los que venían eran delincuentes, personas hacer daño... Nosotras, las mujeres, decidimos arreglar el parque... lo necesitamos todos. El proceso empezó sacando todo lo que hubiera de escombros, dejando el parque limpio y organizándonos más con la Municipalidad para cortar el pasto." (Shirleny Vargas, comunicación personal, marzo 6, 2018)

Es en este punto, se inicia un complemento de capacitación para el comité y el liderazgo comunitario con miras a consolidar una Asociación de Desarrollo Específica y la confección de un proyecto de infraestructura de aprovechamiento del espacio público. Con apoyo de la articulación intersectorial, quienes aportan el financiamiento y por medio de jornadas de construcción, se consolidó el mejoramiento del espacio público con colocación de mobiliario urbano, la instalación de juegos infantiles, siembra de árboles y arbustos como se observa en las Figuras 5 y 6 .

Figura 5: Sesión de "Manos a la Obra" y voluntariado corporativo. Autoría: Asociación Semillas.

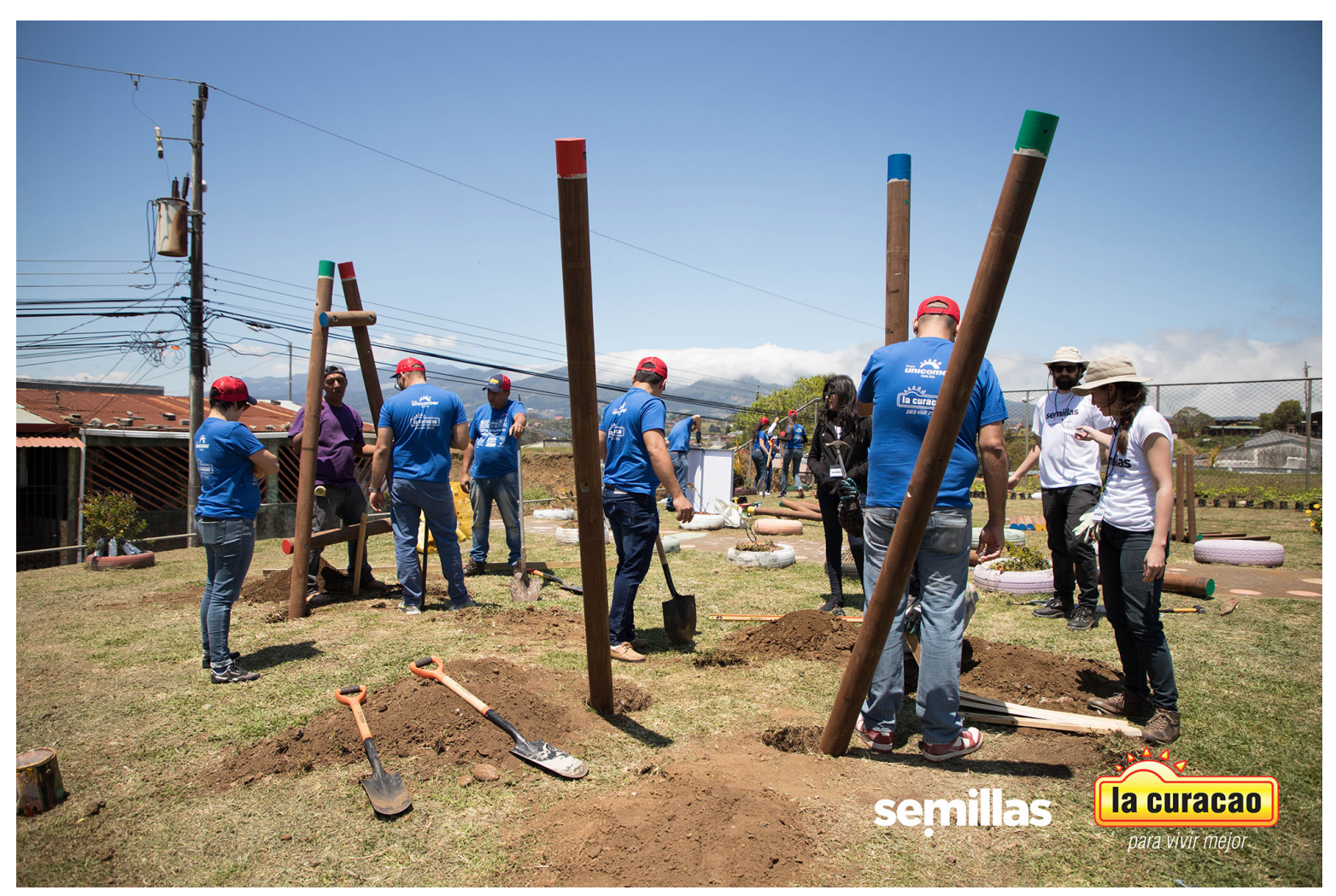

Al igual que en el caso del proceso con las comunidades de Los Olivos y Los Geranios en Paso Ancho, este proyecto se perfila como un proceso compuesto entre el comité pro mejoras del parque y Asociación Semillas, en donde, según explica Camacho, existe un "proceso colaborativo de integración entre las bases comunitarias organizadas, apertura a la participación de nuevos integrantes de la comunidad, representación de instituciones públicas (gobierno local o central), empresas privadas, instituciones académicas, organizaciones no gubernamentales y civiles, entre otras. Se demuestra una clara iniciativa de cambio con respecto a la transformación social y los roles de 
Figura 6: Sesión de "Manos a la Obra". Autoría: Asociación Semillas. poder para actuar en cuanto al desarrollo de proyectos de mejoramiento del espacio habitable. Busca generar vínculos de corresponsabilidad... Se requiere de un equipo de coordinación donde exista representatividad de las partes para que la comunicación sea recíproca y eficaz." . (Silvia Camacho, comunicación personal, junio 21, 2018)

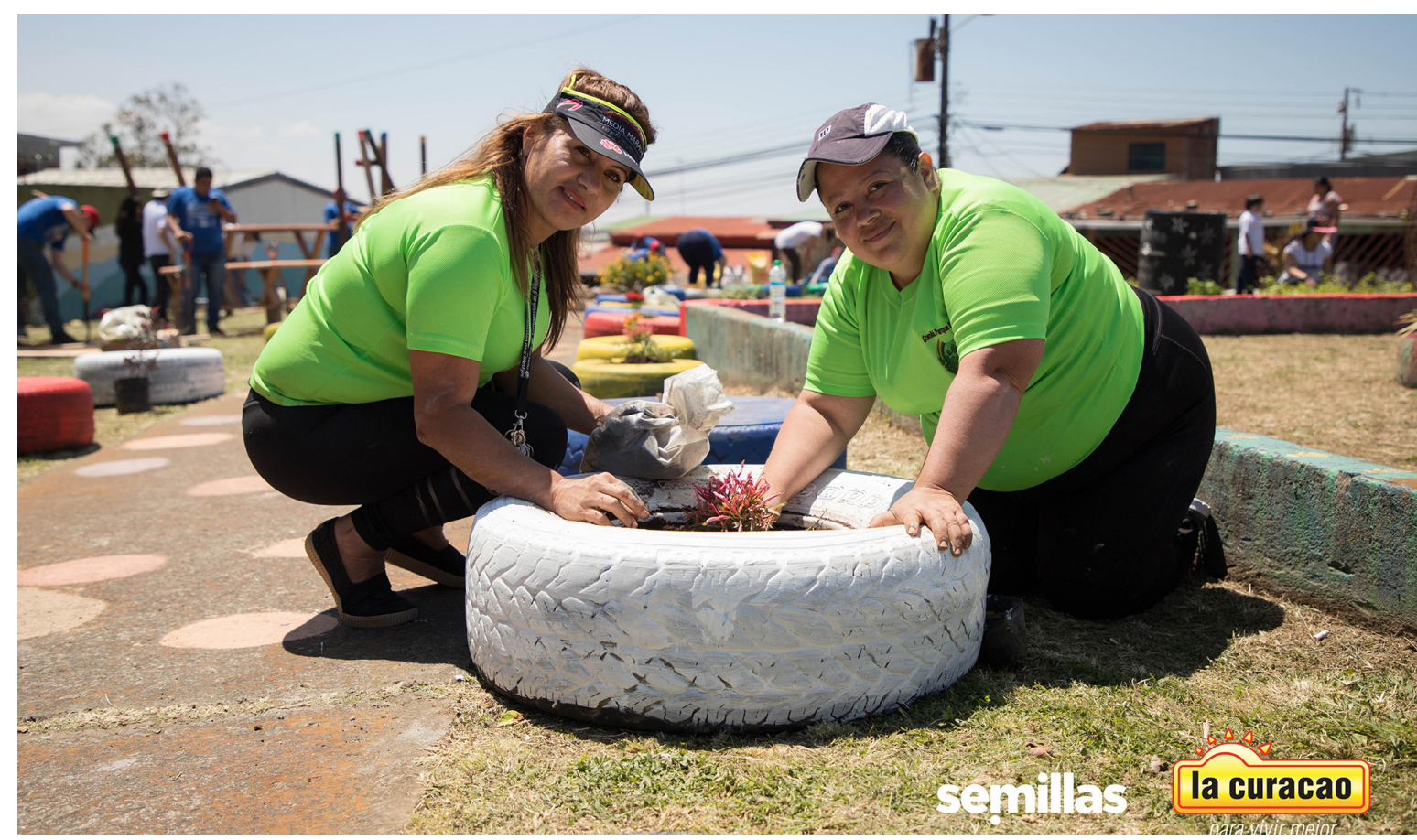

Estos tres procesos anteriormente presentados contribuyen a mostrar cómo se podrían recuperar espacios públicos desde la participación activa como un medio para el desarrollo comunitario. De esta forma, se fomenta una gestión de la ciudad más enfocada al desarrollo impulsado por comunidades (bottom-up). "El desarrollo impulsado por las comunidades surge de organización barrial con el fin de recuperar los intersticios de tejido urbano degradado mediante iniciativas concertadas entre los propietarios y un profesional, a través de procesos de autoconstrucción" (Lira, 2016, p. 36).

\section{Conclusión}

Este ensayo se desarrolló en dos principales secciones. Primeramente, se expuso un planteamiento teórico alrededor de los conceptos de derecho a la ciudad, producción del espacio, participación activa, espacios públicos y la recuperación de estos. Posteriormente, se presentaron tres procesos con comunidades que buscan pasar de la teoría a la práctica.

La ciudad y los espacios públicos se construyen desde la gente y no desde un escritorio. Son las personas que habitan las comunidades quienes día a día construyen su espacio público, pero también el espacio social del mismo por medio de la (re)apropiación y la generación de vivencias en estos espacios. Los espacios públicos no se establecen únicamente por medio de la edificación de infraestructura de aprovechamiento o por medio de un diseño urbano, si no que requiere de la existencia del espacio social y simbólico que promueva el intercambio social, de manera que estos resulten identificables como espacios de convivencia, encuentro y expresión. La participación activa permite el empoderamiento de los habitantes de las ciudades, el desarrollo comunitario, y el fortalecimiento de redes y vínculos vecinales. "El concepto de derecho a la ciudad, incluidas sus reapropiaciones, constituye un valioso repertorio para la investigación social de las transformaciones económicas, culturales, morfológicas, tecnológicas, y políticas urbanas" (Molano, 2016, p. 17).

Es importante que el involucramiento de la comunidad cuente con un grupo representativo con el fin de poder escuchar las diversas opiniones de cada uno y así corroborar que el interés y las necesidades sean verdaderamente de la mayoría. Desde la labor profesional y, especialmente como agentes externos, es vital tener un acercamiento a la comunidad en donde se genere una confianza entre todas las personas participantes. Los procesos sociales son espacios neutros en donde cada uno de los presentes es igual al siguiente, sin importar si es miembro de la comunidad, agente externo, profesional, ama de casa, infante, joven o persona adulta. Son espacios de intercambios de conocimientos y todos tienen algo que aportar. 
La participación activa se perfila como un medio vital para la recuperación de los espacios públicos, para la producción de los espacios y de la ciudad y como un mecanismo para hacer velar el derecho a la ciudad. El marco conceptual planteado en el presente ensayo aporta una perspectiva interconectada de teorías y conceptos que deben considerarse a la hora de pasar a la práctica con procesos sociales participativos de espacio público. Ahora, es importante reflexionar acerca de cómo debe referirse o contemplarse la participación activa en torno a estos abordajes. ¿Será esta una condición individual de los participantes o un reflejo de la totalidad del proceso en sí? Como se expuso en el caso de Los Olivos y Los Geranios, la participación en un procedimiento puede manifestarse de manera variada y en rango cambiante dentro de los habitantes de un mismo proceso. "El espacio público también tiene una dimensión sociocultural. Es un lugar de relación y de identificación, de contacto entre las personas, de animación urbana, y a veces de expresión comunitaria" (Borja y Muxí, 2000, p. 27).

\section{Referencias Bibliográficas}

Awon, N., Schneider, T. \& Till, J. (2011). SpatialAgency. Other ways of doing architecture. Londres: Routledge.

Borja, J. (2012). Espacio Público y Derecho A La Ciudad. Barcelona.

Borja, J., \& Muxí, Z. (2003). El espacio público: ciudad y ciudadanía: Electa.

Camacho, S. (2017). Arquitectura Social Comunitaria. Un diagnóstico de los fenómenos socioespaciales inmersos en la práctica de la "arquitectura social comunitaria" y la metodología del "diseño participativo" para la propuesta de espacios públicos comunitarios en Costa Rica. (Licenciatura en Arquitectura), Universidad de Costa Rica, San José, Costa Rica.

Contraloría General de la República. (2017). Índice de Gestión Municipal. Resultados del período 2016 (Informe DFOE-DL-IF-00007-2017).

Gutiérrez, F. (2006). En Busca del Sentido. Doctorado en Educación para la Tercera Cultura, Universidad La Salle; San José.

Harvey, D. (2014). Seventeen Contradictions and the End of Capitalism: Oxford University Press.

Holston, J. (2008). Insurgent citizenship: Disjunctions of democracy and modernity in Brazil. Nueva Jersey: Princeton University Press.

Lefebvre, H., Lorea, I. M., \& Gutiérrez, E. M. (2013). La producción del espacio: Capitán Swing.

Lira, D. (2016). Gestión de los espacios públicos. Metodología para los procesos sociales desde la perspectiva de hábitat. (Licenciatura en Arquitectura), Universidad de Costa Rica, San José, Costa Rica.

Molano Camargo, F. (2016). El derecho a la ciudad: de Henri Lefebvre a los análisis sobre la ciudad capitalista contemporánea. Folios, 2(44), 3-19.

Morales Alpízar, M., \& Obando Márquez, L. M. (2016). Cogestionando el Hábitat en Comunidades Aprendientes con Habitantes de Asentamientos Informales Urbanos. Hacia una Pedagogía del Hábitat. (Doctorado en Educación para la Tercera Cultura (informe preliminar)), Universidad de La Salle.

Municipalidad de San José (2015). Política de Participación y Corresponsabilidad Ciudadana-PPCC-.Recuperadode:https://www.msj.go.cr/informacion_ciudadana/ SiteAssets/Planificaci \%C3\%B3 n\%20institucional/Pol\%C3\%ADtica\%20de\%20 Participaci\%C3\%B3n\%20y\%20Corresponsabilidad\%20Ciudadana\%20-PPCC-. pdf

Suárez, S. (2018). Laboratorio Urbano Abierto. Producción colectiva del espacio urbano a través de exploraciones socio-virtuales del entorno. (Licenciatura en Arquitectura), Universidad de Costa Rica, San José, Costa Rica. 